\title{
CLUSTERS WITH EXTENDED RADIO EMISSION AT HIGH FREQUENCIES
}

\author{
R. Wielebinski \\ Max-Planck-Institut für Radioastronomie, Bonn, F.R.G.
}

The existence of 'haloes' in clusters of galaxies was deduced by Ryle and Windram (1968) for the Perseus cluster and by Willson (1970) for the Coma cluster at $408 \mathrm{MHz}$ by comparing total flux measured by a single dish with the sum of fluxes of radio sources found in the field. A direct measurement of the extended source Coma $C$ was made by Jaffe et al. (1976) at $610 \mathrm{MHz}$. The failure to detect the halo of Coma at higher frequencies is attributed by all authors to a steep spectrum of this extended component.

The 100-m radio telescope of the MPIfR was used successfulity to map normal galaxies with $3 \mathrm{mK}$ r.m.s. noise at $2.7 \mathrm{GHz}$ and $1.5 \mathrm{mK}$ at $4.8 \mathrm{GHz}$. These noise levels are still slightly higher than the confusion 1 imit of the telescope and are ideally suited to map low-luminosity extended regions. To overcome problems of dynamic range limitation a sidelobe cleaning procedure was developed which allows the recognition of features $30 \mathrm{~dB}$ below a strong source.

The survey of clusters of galaxies at $2.7 \mathrm{GHz}$ contains some 30 clusters, all suspected to have extended emission on the scale $>5^{\prime}$. Positive detections of extended emission could so far be reported for A754, A2142, A2256 and A2319 (Haslam et a1. 1977). The extended emission does not permeate the whole cluster volume, but is usually present near the dominant galaxies. All these clusters are known to be X-ray sources. On the other hand, most of the clusters reported to be extended by Owen (1974) have been resolved into individual radio sources. Clusters for which our 4.6 beam at $2.7 \mathrm{GHz}$ gives not sufficient angular resolution are being mapped at $4.8 \mathrm{GHz}$ with a $2: 6$ beam.

Haslam, C.G.T., Kronberg, P.P., Waldthausen, H., Wielebinski, R., Schallwich, D. 1977, Astron. Astrophys. Suppl. (in press) Jaffe, W.J., Perola, G.C., Valentijn, E.A. 1976. Astron. Astrophys. 49,179

Ryle, M., Windram, M.D. 1968, Monthly Notices Roy. Astron. Soc. 138, 1 Willson, M.A.G. 1970, Monthly Notices Roy. Astron. Soc. 151, 1 


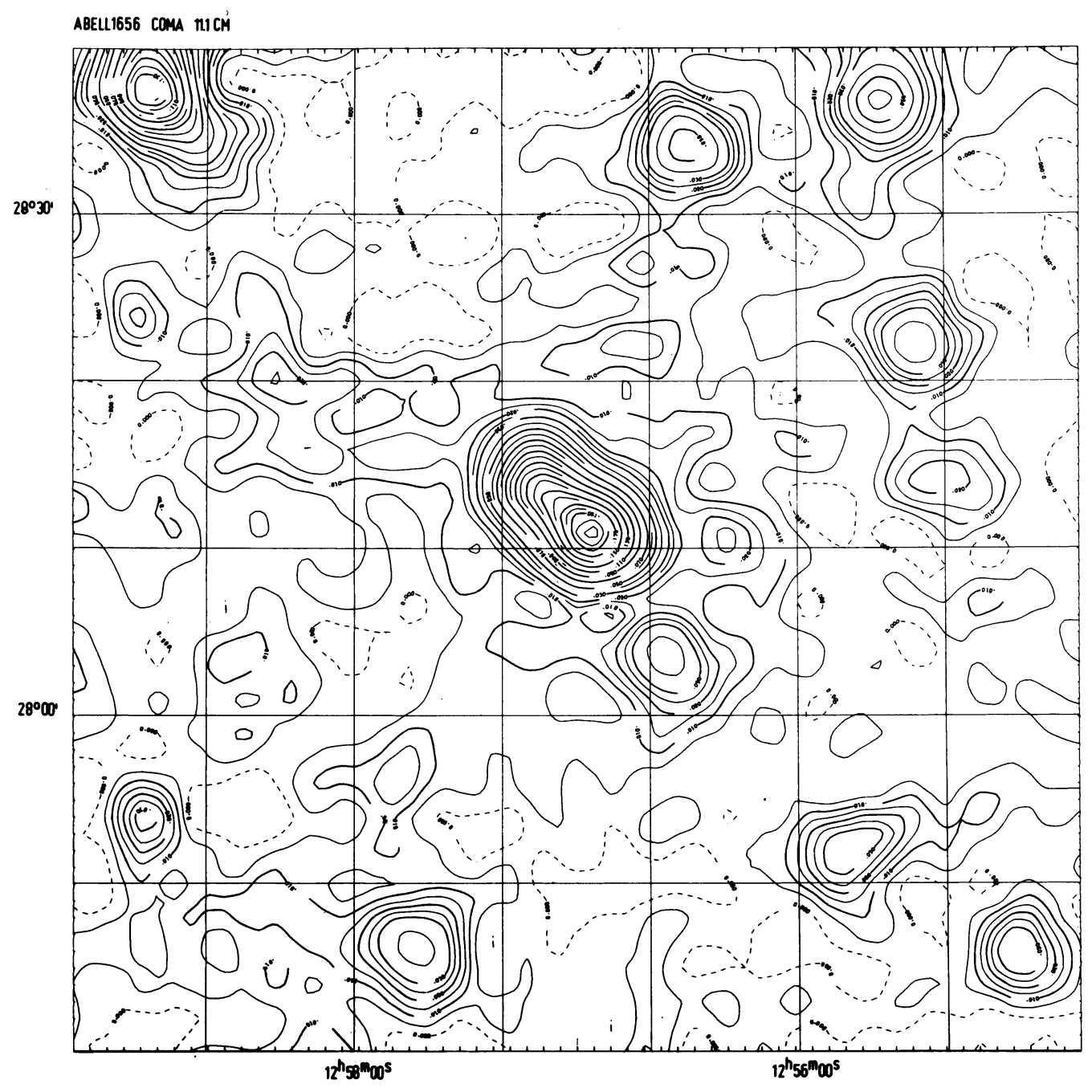

An $11.1 \mathrm{~cm}$ map of COMA (Abe11 1656). The contours are $0,5,10, \ldots$ $30,40, \ldots 210 \mathrm{mJy} / \mathrm{beam}$ area. Beamwidth is 4.6 arc min to $3 \mathrm{~dB}$ points. Note $5 \mathrm{mJy} /$ beam area $=10 \mathrm{mK} \mathrm{T}_{\mathrm{b}}$. Note extended emission is seen to surround the sources $5 \mathrm{C} 4.85$ and $5 \mathrm{C} 4.81$ which are merged due to the beam and steep spectrum of the tailed source. The extent of "the halo" is some 15 arc min, which corresponds to a linear size of $\sim 500 \mathrm{kpc}$ assuming the distance of Coma to be $\sim 100 \mathrm{Mpc}$. 


\section{DISCUSSION}

Silk: Is there any tendency for X-ray clusters to possess extended radio emission generally?

Wielebinski: So far all the extended radio sources detected are also $\mathrm{X}$-ray sources. But the statistics are still poor and we must await correlations between various parameters, e.g. richness, to see which correlation is unique.

Tarenghi: Is the bridge between NGC 1265 and NGC 1275 real?

Wielebinski: No. This slide shows a simple addition of several maps. Sidelobes give the effect of a bridge. Using sidelobe removal a new map of Perseus cluster is now being made. Still the conclusion about the absence of a large Perseus halo is suggested by the present map. 